\title{
Reactor antineutrino spectra and their application to antineutrino-induced reactions. II
}

\author{
P. Vogel and G. K. Schenter \\ California Institute of Technology, Pasadena, California 91125 \\ F. M. Mann and R. E. Schenter \\ Hanford Engineering Development Laboratory, Richland, Washington 99352
}

(Received 29 January 1981)

\begin{abstract}
The antineutrino and electron spectra associated with various nuclear fuels are calculated. While there are substantial differences between the spectra of different uranium and plutonium isotopes, the dependence on the energy and flux of the fission-inducing neutrons is very weak. The resulting spectra can be used for the calculation of the antineutrino and electron spectra of an arbitrary nuclear reactor at various stages of its refueling cycle. The sources of uncertainties in the spectrum are identified and analyzed in detail. The exposure time dependence of the spectrum is also discussed. The averaged cross sections of the inverse neutron $\beta$ decay, weak charged and neutral-current-induced deuteron disintegration, and the antineutrino-electron scattering are then evaluated using the resulting $\bar{v}_{e}$ spectra.
\end{abstract}

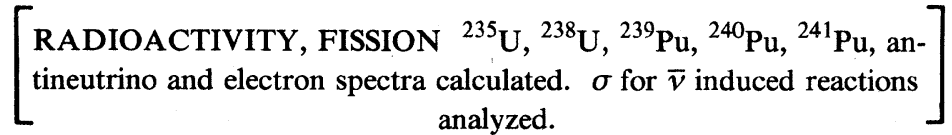

\section{INTRODUCTION}

New and accurate results, with potentially far reaching consequences, have recently become available in the field of reactor neutrino physics. The deuteron disintegration by reactor antineutrinos indicates neutrino instability. ${ }^{1}$ Accurate measurement of the inverse neutron beta decay, ${ }^{2}$ on the other hand, does not imply the existence of neutrino oscillations with parameters compatible with the results of Ref. 1 . These results, and related ones on neutrino mass ${ }^{3}$ and on possible evidence for neutrino oscillations ${ }^{4}$ at high energies, have motivated our present work.

One of the more serious problems in interpreting the results of experiments with reactor antineutrinos is the uncertainty in the spectrum of antineutrinos emerging from the reactor. This problem was studied in our previous work, ${ }^{5}$ which hereafter will be referred to as I. Since its publication several other papers on the subject have been published as well. The neutrino and electron spectra of A vignone and collaborators ${ }^{6,7}$ are substantially harder than those predicted in $\mathbf{I}$. The calculations in Refs. 8-10 also predict relatively hard spectra. All calculations can be directly tested in measurements of electrons accompanying fission. There has been a new development in this area also. Based on recent electron spectrum measurements at Oak Ridge, Dickens ${ }^{11}$ calculated an antineutrino spectrum virtually identical to that of $\mathrm{I}$. The measured electron spectrum of Schreckenbach and collaborators $^{12}$ also agrees with the prediction of $I$ and disagrees with the results of Refs. 6-10.

The goal of the present work is a generalization of the results presented in I. A new set of experimental beta decay data has been added and additional analyses of the approximations performed. The uncertainty analysis is also more complete and the various sources of error are discussed in detail. One of the outstanding problems is the variation of the antineutrino flux related to the different fuel composition in different reactors 
(and at different times after refueling in the same reactor). To solve this problem we calculate the spectra corresponding to various fissioning nuclei, to various initial neutron energies, and to various neutron fluxes in the reactor. The question of exposure time dependence, of crucial importance when comparing the calculated and experimental electron spectra, is also addressed. Finally, a complete set of predicted averaged cross sections of the most commonly studied antineutrinoinduced reactions is given and discussed.

\section{CALCULATION OF THE SPECTRA}

As in I we use the summation method in the calculation of the antineutrino spectrum, that is

$$
\begin{aligned}
N\left(E_{\bar{v}}\right)= & \sum_{n} Y_{n}(Z, A, t) \\
& \times \sum_{i} b_{n, i}\left(E_{0}^{i}\right) P_{\bar{v}}\left(E_{\bar{v}}, E_{0}^{i}, Z\right) .
\end{aligned}
$$

Here $Y_{n}(Z, A, t)$ is the number of beta decays per unit time of the fragment $Z, A$ (possibly isomeric state) after the fissioning material has been exposed to neutrons for a time $t$. For $t$ larger than the beta decay lifetime of the fragment $Z, A$ the quantity $Y_{n}$ converges toward the cumulative fission yield and becomes independent of $t$. Naturally, a different set of $Y_{n}$ values has to be used for each actinide nucleus and for each incident neutron energy. Generally $Y_{n}$ does depend on $t$ and should include the effect of transmutations of fission fragments by reactor neutrons and of the delayed neutron emission. In our calculations we have used the cumulative yields of the set

ENDF/B-V (Ref. 13) nuclear data library (Tables I and II) and also the time dependent solutions of the coupled system of differential equations describing fission, subsequent beta decay, and possible neutron capture by the fission fragments (see Fig. 4 and the discussion of the neutron flux effects).

TABLE I. Antineutrino spectra of various actinide nuclei, total numbers of $\bar{v}$ for different groups of nuclei, average electron kinetic energy, and average antineutrino energy. Calculations for the equilibrium situation, i.e., infinite expo-

\begin{tabular}{|c|c|c|c|c|c|c|c|c|c|}
\hline $\begin{array}{c}\text { Nucleus } \\
n \text { energy }(\mathrm{MeV})\end{array}$ & $\begin{array}{l}{ }^{235} \mathrm{U} \\
\text { ther }\end{array}$ & $\begin{array}{l}{ }^{235} \mathrm{U} \\
0.5\end{array}$ & ${ }_{14}^{238} U$ & $\begin{array}{c}{ }^{238} \mathbf{U} \\
0.5\end{array}$ & ${ }_{14}^{238} \mathrm{U}$ & $\begin{array}{l}{ }^{239} \mathrm{Pu} \\
\text { ther }\end{array}$ & $\begin{array}{c}{ }^{239} \mathrm{Pu} \\
0.5\end{array}$ & $\begin{array}{l}{ }^{240} \mathrm{Pu} \\
0.5\end{array}$ & $\begin{array}{l}{ }^{241} \mathrm{Pu} \\
\text { ther }\end{array}$ \\
\hline \multicolumn{10}{|l|}{$E_{\bar{v}}$} \\
\hline 1.0 & 2.44 & 2.44 & 2.17 & 2.77 & 2.59 & 2.34 & 2.35 & 2.47 & 2.64 \\
\hline 1.5 & 1.73 & 1.72 & 1.43 & 1.99 & 1.80 & 1.50 & 1.50 & 1.61 & 1.76 \\
\hline 2.0 & 1.28 & 1.28 & 1.04 & 1.51 & 1.35 & 1.08 & 1.09 & 1.19 & 1.33 \\
\hline 2.5 & $8.60(-1)^{\mathrm{a}}$ & $8.50(-1)$ & $6.80(-1)$ & 1.06 & $9.40(-1)$ & $7.10(-1)$ & $7.14(-1)$ & $7.98(-1)$ & $9.13(-1)$ \\
\hline 3.0 & $5.80(-1)$ & $5.90(-1)$ & $4.60(-1)$ & $7.60(-1)$ & $6.70(-1)$ & $4.77(-1)$ & $4.83(-1)$ & $5.42(-1)$ & $6.32(-1)$ \\
\hline 3.5 & $4.10(-1)$ & $4.10(-1)$ & $3.20(-1)$ & $5.50(-1)$ & $4.80(-1)$ & $3.14(-1)$ & $3.19(-1)$ & $3.61(-1)$ & $4.31(-1)$ \\
\hline 4.0 & $2.82(-1)$ & $2.84(-1)$ & $2.13(-1)$ & $3.86(-1)$ & $3.37(-1)$ & $2.03(-1)$ & $2.07(-1)$ & $2.36(-1)$ & $2.88(-1)$ \\
\hline 4.5 & $1.81(-1)$ & $1.84(-1)$ & $1.34(-1)$ & $2.58(-1)$ & $2.23(-1)$ & $1.21(-1)$ & $1.24(-1)$ & $1.42(-1)$ & $1.79(-1)$ \\
\hline 5.0 & $1.18(-1)$ & $1.20(-2)$ & $8.60(-2)$ & $1.71(-1)$ & $1.47(-1)$ & $7.46(-2)$ & $7.68(-2)$ & $8.82(-2)$ & $1.14(-1)$ \\
\hline 5.5 & $7.20(-2)$ & $7.40(-2)$ & $5.20(-2)$ & $1.06(-1)$ & $9.20(-2)$ & $4.37(-2)$ & $4.52(-2)$ & $5.19(-2)$ & $6.82(-2)$ \\
\hline 6.0 & $4.08(-2)$ & $4.23(-2)$ & $3.04(-2)$ & $6.05(-2)$ & $5.45(-2)$ & $2.37(-2)$ & $2.49(-2)$ & $2.80(-2)$ & $3.71(-2)$ \\
\hline 6.5 & $1.95(-2)$ & $2.07(-2)$ & $1.48(-2)$ & $3.15(-2)$ & $2.90(-2)$ & $1.01(-2)$ & $1.11(-2)$ & $1.23(-2)$ & $1.73(-2)$ \\
\hline 7.0 & $1.04(-2)$ & $1.12(-2)$ & $7.80(-3)$ & $1.74(-2)$ & $1.65(-2)$ & $4.93(-3)$ & $5.54(-3)$ & $6.19(-3)$ & $8.87(-3)$ \\
\hline 7.5 & $5.06(-3)$ & $5.56(-3)$ & $3.53(-3)$ & $9.28(-3)$ & $8.96(-3)$ & $2.20(-3)$ & $2.55(-3)$ & $2.85(-3)$ & $4.49(-3)$ \\
\hline 8.0 & $2.04(-3)$ & $2.40(-3)$ & $1.21(-3)$ & $4.69(-3)$ & $4.57(-3)$ & $7.78(-4)$ & $9.26(-4)$ & $1.11(-3)$ & $2.16(-3)$ \\
\hline$N_{\bar{v}}$ (known) & 4.98 & 4.92 & 4.34 & 5.03 & 4.71 & 4.36 & 4.34 & 4.48 & 4.61 \\
\hline$N_{\bar{v}}$ (Aleklett) & 0.31 & 0.33 & 0.25 & 0.42 & 0.41 & 0.18 & 0.17 & 0.23 & 0.30 \\
\hline$N_{\bar{v}}$ (unknown) & 0.85 & 0.89 & 0.82 & 1.63 & 1.47 & 1.04 & 1.07 & 1.22 & 1.51 \\
\hline$N_{\bar{v}}($ total $)$ & 6.14 & 6.14 & 5.41 & 7.08 & 6.59 & 5.58 & 5.58 & 5.93 & 6.42 \\
\hline$\left\langle T_{e}\right\rangle(\mathrm{MeV} /$ fiss $)$ & 6.5 & & & 8.1 & & 5.2 & & & 6.6 \\
\hline$\left\langle E_{\bar{v}}\right\rangle(\mathrm{MeV} /$ fiss $)$ & 9.0 & & & 11.1 & & 7.4 & & & 9.3 \\
\hline
\end{tabular}
sure time. Spectra are in units of $\bar{v} / \mathrm{MeV}$ fiss, $N$ 's in $\bar{v} /$ fiss, and average energies in $\mathrm{MeV} /$ fiss.

a $8.60(-1)$ denotes $8.60 \times 10^{-1}$. 
TABLE II. Electron spectra. (See caption of Table I.)

\begin{tabular}{|c|c|c|c|c|c|c|c|c|c|}
\hline $\begin{array}{c}\text { Nucleus } \\
n \text { energy }(\mathrm{MeV})\end{array}$ & $\begin{array}{l}{ }^{235} \mathrm{U} \\
\text { ther }\end{array}$ & $\begin{array}{c}{ }^{235} \mathrm{U} \\
0.5\end{array}$ & ${ }_{14}^{235} \mathrm{U}$ & $\begin{array}{c}{ }^{238} \mathrm{U} \\
0.5\end{array}$ & ${ }_{14}^{238} \mathrm{U}$ & $\begin{array}{l}{ }^{239} \mathrm{Pu} \\
\text { ther }\end{array}$ & $\begin{array}{c}{ }^{239} \mathrm{Pu} \\
0.5\end{array}$ & $\begin{array}{c}{ }^{240} \mathrm{Pu} \\
0.5\end{array}$ & $\begin{array}{l}{ }^{241} \mathrm{Pu} \\
\text { ther }\end{array}$ \\
\hline \multicolumn{10}{|l|}{$E_{e-}^{\text {tot }}$} \\
\hline 1.0 & 3.09 & 3.09 & 2.74 & 3.46 & 3.24 & 2.95 & 2.95 & 3.11 & 3.31 \\
\hline 1.5 & 2.01 & 2.01 & 1.69 & 2.31 & 2.10 & 1.77 & 1.77 & 1.89 & 2.06 \\
\hline 2.0 & 1.35 & 1.34 & 1.09 & 1.59 & 1.43 & 1.14 & 1.15 & 1.25 & 1.39 \\
\hline 2.5 & $8.80(-1)$ & $8.80(-1)$ & $7.10(-1)$ & 1.09 & $9.69(-1)$ & $7.35(-1)$ & $7.39(-1)$ & $8.20(-1)$ & $9.36(-1)$ \\
\hline 3.0 & $5.86(-1)$ & $5.90(-1)$ & $4.64(-1)$ & $7.55(-1)$ & $6.68(-1)$ & $4.72(-1)$ & $4.78(-1)$ & $5.36(-1)$ & $6.24(-1)$ \\
\hline 3.5 & $3.96(-1)$ & $3.98(-1)$ & $3.09(-1)$ & $5.26(-1)$ & $4.64(-1)$ & $3.03(-1)$ & $3.08(-1)$ & $3.47(-1)$ & $4.13(-1)$ \\
\hline 4.0 & $2.65(-1)$ & $2.68(-1)$ & $2.01(-1)$ & $3.61(-1)$ & $3.17(-1)$ & $1.89(-1)$ & $1.94(-1)$ & $2.20(-1)$ & $2.68(-1)$ \\
\hline 4.5 & $1.72(-1)$ & $1.74(-1)$ & $1.28(-1)$ & $2.40(-1)$ & $2.10(-1)$ & $1.15(-1)$ & $1.18(-1)$ & $1.34(-1)$ & $1.68(-1)$ \\
\hline 5.0 & $1.08(-1)$ & $1.11(-1)$ & $7.99(-2)$ & $1.55(-1)$ & $1.35(-1)$ & $6.84(-2)$ & $7.06(-2)$ & $8.06(-2)$ & $1.03(-1)$ \\
\hline 5.5 & $6.55(-2)$ & $6.70(-2)$ & $4.82(-2)$ & $9.49(-2)$ & $8.36(-2)$ & $3.95(-2)$ & $4.11(-2)$ & $4.67(-2)$ & $6.04(-2)$ \\
\hline 6.0 & $3.69(-2)$ & $3.81(-2)$ & $2.76(-2)$ & $5.43(-2)$ & $4.90(-2)$ & $2.10(-2)$ & $2.23(-2)$ & $2.50(-2)$ & $3.27(-2)$ \\
\hline 6.5 & $1.92(-2)$ & $2.02(-2)$ & $1.46(-2)$ & $2.96(-2)$ & $2.74(-2)$ & $1.01(-2)$ & $1.10(-2)$ & $1.23(-2)$ & $1.66(-2)$ \\
\hline 7.0 & $9.66(-3)$ & $1.03(-2)$ & $7.16(-3)$ & $1.58(-2)$ & $1.50(-2)$ & $4.55(-3)$ & $5.10(-3)$ & $5.68(-3)$ & $8.11(-3)$ \\
\hline 7.5 & $4.68(-3)$ & $5.10(-3)$ & $3.28(-3)$ & $8.38(-3)$ & $8.12(-3)$ & $2.02(-3)$ & $2.32(-3)$ & $2.61(-3)$ & $4.04(-3)$ \\
\hline 8.0 & $2.05(-3)$ & $2.37(-3)$ & $1.28(-3)$ & $4.32(-3)$ & $4.26(-3)$ & $7.79(-4)$ & $9.23(-4)$ & $1.09(-3)$ & $1.93(-3)$ \\
\hline
\end{tabular}

The quantities $b_{n, i}\left(E_{0}^{i}\right)$ in Eq. (1) are branching ratios for the $i$ th branch with the maximal electron energy $E_{0}^{i}=Q_{n}+m_{e} c^{2}-E_{\mathrm{exc}}^{i}$, where $Q_{n}$ is the beta decay $Q$ value of the fission fragment $n$, and $E_{\text {exc }}^{i}$ is the excitation energy in the daughter nu- cleus. The branching ratios are normalized to unity except for isomeric states, where the same is smaller due to the $\gamma$ decay.

As in I we assume that all branches have the allowed spectral shape, i.e.,

$$
P_{\bar{v}}\left(E_{\bar{v}}, E_{0}, Z\right)=k\left(E_{0}, Z\right) E_{\bar{v}}{ }^{2}\left(E_{0}-E_{\bar{v}}\right)\left[\left(E_{0}-E_{\bar{v}}\right)^{2}-m_{e} c^{2}\right]^{1 / 2} F\left(E_{0}-E_{\bar{v}}, Z\right),
$$

where $k$ is the normalization constant. The function $F$ is the usual Fermi Coulomb function; as in I we usually used a simple analytic approximation to it, without appreciable loss of accuracy.

When calculating the associated electron spectrum, the only modification needed is the spectrum shape factor $P_{\bar{v}}$ which is now replaced by

$$
\begin{aligned}
P_{c}\left(E_{\beta}, E_{0}, Z\right)= & k\left(E_{0}, Z\right)\left(E_{0}-E_{\beta}\right)^{2} \\
& \times E_{\beta} p_{\beta} F\left(E_{\beta}, Z\right) .
\end{aligned}
$$

Both the electron and antineutrino spectra are calculated together and depend in approximately the same way on the input parameters.

In order to evaluate Eq. (1) one has to know all the necessary values of $Y_{n}, Q_{n}$, and $b_{n, i}$. It is convenient and instructive to divide all these quantities into two sets: "yield" data, that is, the $Y_{n}$ values, and "decay" data (that is, everything else). Note that the same set of decay data describes the fission of all actinide nuclei with all incident neutron energies. Consequently, the differences between the corresponding spectra are more certain than the absolute values of each spectrum separately.

The decay data of ENDF/B-V (Ref. 13) were used in our calculation. This file contains 744 unstable fission products (including isomers) of which 269 have experimentally complete decay schemes. These "known" nuclei contribute 
$75-85 \%$ of the total beta decay yield (see Table I).

The procedure used for the "unknown" nuclei was the same as in I; their role here is, however, somewhat smaller, because new data have been added to the decay file. Briefly, the following steps were taken:

(a) The $Q$ values were checked. For about 20 fission products with non-negligible fission yields the $Q$ values in ENDF/B-V are based on systematic or semiempirical mass relations. ${ }^{14,15}$ The effect of this approximation is discussed in Sec. III.

(b) For about 40 nuclei the continuous $\beta$-feed distribution of Aleklett et al. ${ }^{16}$ were used. The number of such nuclei is smaller than in I, because some of them became known in the meantime. The total yield of these nuclei is shown in Table I and their relative contribution to the spectrum is shown in Fig. 1.

(c) For the remaining unknown nuclei the model of constant reduced transition rate, as in $I$, has been adopted. Thus

$$
\left.b\left(E_{0}\right)=k^{\prime} \rho\left(Q+m_{e} c^{2}-E_{0}\right) \int_{m_{e} c^{2}}^{E_{0}}\left(E_{0}-E_{\beta}\right)^{2} p_{\beta} E_{\beta} F\left(E_{\beta}, Z+1\right) d E_{\beta}\right)
$$

where $k^{\prime}$ is the normalization constant and $\rho\left(E_{\text {exc }}\right)$ is the nuclear level density. The branching ratios of Eq. (4) are normalized by the condition

$$
\sum_{E_{\mathrm{exc}}>p} b\left(E_{0}\right)=\alpha^{\prime}
$$

Here $P$ is the daughter nucleus pairing energy and $\alpha^{\prime}$ is the total feeding to states above the pairing gap. The parameter $\alpha^{\prime}$ is the "free" parameter of the model and it was found by averaging the results of Ref. 16. We use, as in $I, \alpha^{\prime}=1$ for odd-odd daughters, $\alpha^{\prime}=0.53$ for nuclei with $Q$ $P \geq 5 \mathrm{MeV}$ and $A<110, \alpha^{\prime}=0.36$ for $Q-P \geq 5$ $\mathrm{MeV}$ and $A \geq 110$, and $\alpha^{\prime}=0.28$ for the remaining nuclei. From the distribution of $\alpha^{\prime}$ in Ref. 16

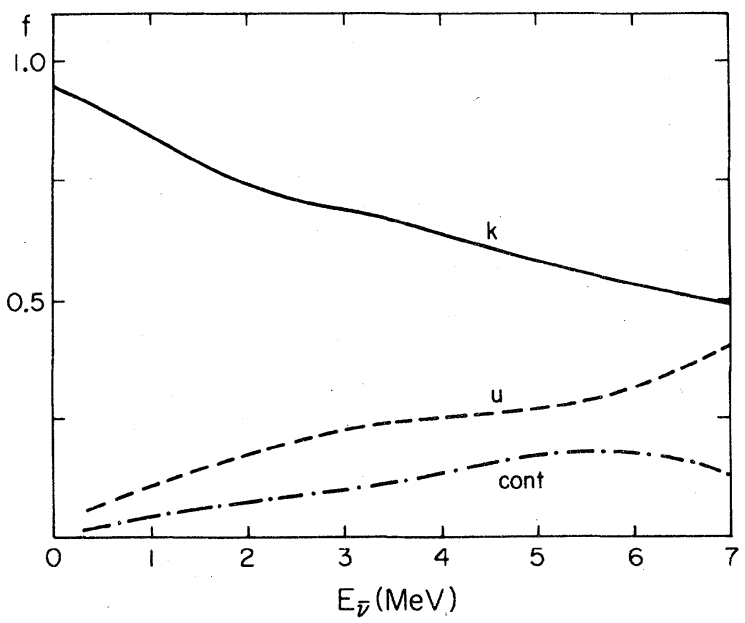

FIG. 1. Contribution of the known $(k)$, unknown $(u)$, and on line (cont) nuclei to the antineutrino spectrum of fissioning ${ }^{235} \mathrm{U}$ at various energies. we assign $50 \%$ uncertainty to $\alpha^{\prime}$ for the second and third groups, and $80 \%$ for the last group. The remaining 1- $\alpha^{\prime}$ branching was equally distributed among three hypothetical states with energies $0, P / 3$, and $2 P / 3$. Let us stress that this adopted prescription generally gives more feeding to high lying states than the prescriptions adopted in Refs. 6 and 7 (and in previous calculations of the same group) or in Refs. 8-10.

The motivation for this procedure was discussed in I. We realize that our description of the beta decay of unknown nuclei is still somewhat primitive and that perhaps microscopic calculations, as developed for example by Klapdor and Wene, ${ }^{17}$ better describe the beta decay of individual fission fragments; however, in our case only the averaged behavior of a large group of nuclei is needed and our simpler phenomenological approach seems adequate (see Fig. 2).

The resulting spectra of antineutrinos and electrons (note that we use the total electron energy in column 1 of Table II), are shown in Tables I and II for a variety of fission fuels. Table I also lists some integral characteristics of the spectra. The yields used in calculating these spectra are cumulative yields, which include the delayed neutron effect. Thus this calculation corresponds to the "equilibrium" or infinite exposure time situation.

\section{ANALYSIS OF THE SPECTRUM}

In this section we discuss the approximations, the uncertainties, and the dependence of spectrum on the exposure time, reactor neutron flux, etc. The relative contribution to the ${ }^{235} \mathrm{U}$ antineutrino spectrum from the three categories of nuclei described 


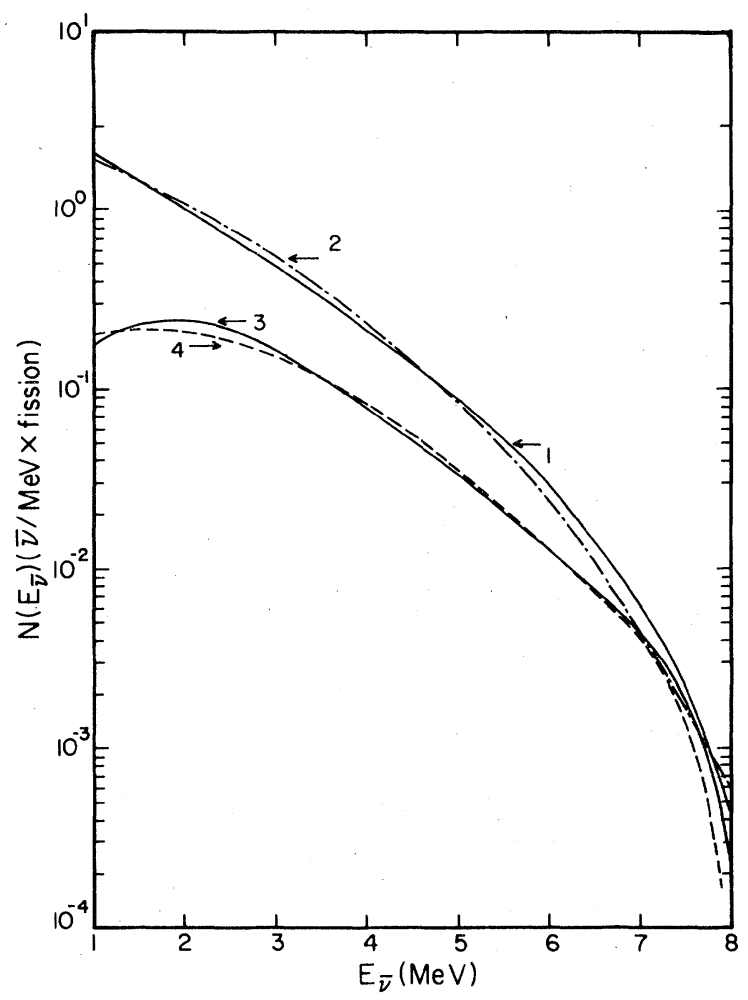

FIG. 2. Tests of model assumptions for branching ratios. Curve 1 is the spectrum corresponding to all nuclei in the experimentally known decay branches. Curve 2 uses the same set of nuclei but model branching ratios are used instead of the experimental ones. Curves 3 and 4 show spectra of 23 nuclei where both "line" and "continuous" spectra are available. Curve 3 uses the line spectra, and curve 4 uses the continous spectra of Ref. 16.

above is shown in Fig. 1. The "on line" data of Ref. 16 are used in our calculations in two ways. First, some nuclei were experimentally studied only in this way and their continuous beta feed functions are used directly (curve "cont" in Fig. 1). Secondly, the experience of Ref. 16 was used in the determination of the prescription for the unknown nuclei [Eq. (4)] and in determination of the parameter $\alpha^{\prime}$ [Eq. (5)].

Twenty-three short lived isotopes were studied not only in Ref. 16 but also by standard beta spectroscopy. The corresponding antineutrino spectra of these nuclei, weighted by their cumulative fission yields in ${ }^{235} \mathrm{U}$, are shown in Fig. 2 (curves 3 and 4). The good agreement between them shows that the two experimental techniques are consistent with each other.

We noted above that the results of Ref. 16 were used in deriving the prescription for treatment of nuclei with the unknown decay schemes. It is interesting to see how well this prescription describes the nuclei with known decay schemes. Such a comparison is also shown in Fig. 2 (curves 1 and 2). In calculating curve 2 we used the subset of all 269 known nuclei with their yields and $Q$ values, but we replaced the experimental branching ratios by the model ones. Naturally, these two sets of branching ratios differ in individual nuclei. However, the averaging over a large set of nuclei leads to the good agreement between the two spectra. Note that no parameter fitting is involved.

Now we approach the problem of error analysis. The uncertainties in the resulting electron and antineutrino spectra consist of contributions from four independent sources, related to the uncertainties in the fission yields [or more generally the $Y_{n}$ values in Eq. (1)], in the beta decay $Q$ values, in the individual branching ratios, and to uncertainties related to our model assumptions. We neglect an additional source of error, the possibility that some beta decays do not have the allowed spectral shape.

The fission yield errors for different mass chains ( $A$ values) are independent. Within a given $A$ chain the cumulative yields are to some degree correlated, but such correlations are not very important. In

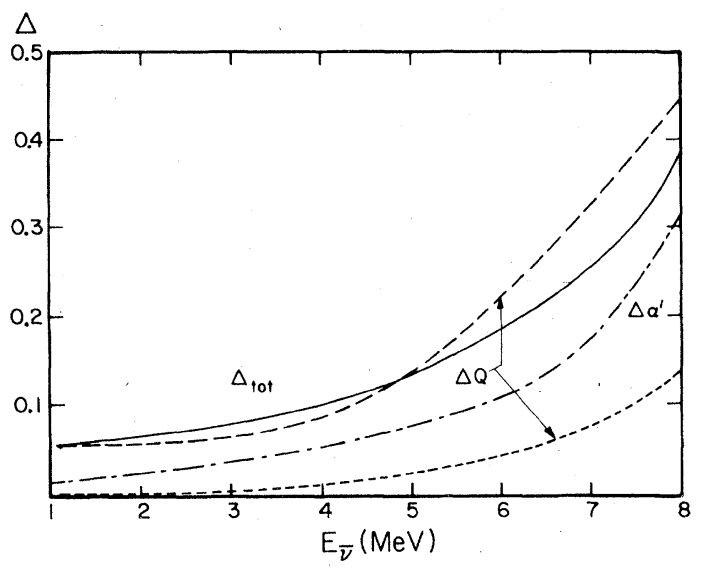

FIG. 3. The relative spectrum uncertainties at different antineutrino energies. The dashed curves show the uncertainties associated with the beta decay $Q$ values; the lower curve corresponds to independent errors, the upper curve to fully correlated errors. The dot-and-dashed curve shows the uncertainties associated with the parameter $\alpha^{\prime}$, Eq. (5). The full curve shows the total estimated uncertainty. 
any case, the error in the spectrum associated with the fission yield uncertainties is quite small, less than $2 \%$ when individual errors are added in quadratures. Even when the errors are treated as fully correlated, they give only $4 \%$ at low energies and about $10 \%$ at $8 \mathrm{MeV}$.

It is more difficult to estimate the uncertainties related to the $Q$ values. We used the experimental $\Delta Q$ values whenever possible, otherwise we used a "standard" value $\Delta Q=0.3 \mathrm{MeV}$ for $Q<5 \mathrm{MeV}$ and $\Delta Q=0.6 \mathrm{MeV}$ for $Q>5 \mathrm{MeV}$. The crucial problem, of course, is the correlation between these errors. As remarked before some of the largest $Q$ values are not based on measurements but are deduced from nuclear mass formulas. Such $Q$ values have obviously correlated uncertainties. In Fig. 3 we show the lower and upper limits of uncertainties associated with $\Delta Q$, obtained by adding the errors in quadrature (lower curve) or in absolute values (upper curve). Neither of these is quite realistic, but in our opinion the true error is closer to the lower curve.

In this context a following point should be made. Recent measurements of masses of neutron rich alkali metals ${ }^{18}$ suggest that the formula of Jänecke ${ }^{15}$ results in an underestimate of the corresponding masses. This formula has been used in our work when experimental $Q$ values were not available. (This problem was pointed out in Ref. 1). To estimate the associated error we replaced the corresponding $Q$ values by predictions based on the Liran-Zeldes mass formula, ${ }^{15}$ which agrees better with the alkali metal masses of Ref. 18. The average $Q$ value for the affected nuclei is increased by about $0.6 \mathrm{MeV}$ (hence the standard $\Delta Q$ ) and the antineutrino spectrum is practically unchanged below $5 \mathrm{MeV}$, and is increased by about $6 \%(\sim 12 \%)$ at 6 $\mathrm{MeV}(8 \mathrm{MeV})$. This change is well within the uncertainties and thus we decided to keep the originally assigned $Q$ values.

Somewhat similar comments can be made about errors related to the uncertainties in individual branching ratios. In our treatment we used the experimental $\Delta b$ values when they were available, and a standard value $\Delta b / b=0.2$ otherwise. When such errors are treated as independent the resulting total error is again quite small, changing from about $1 \%$ at low energies to about $6 \%$ at $8 \mathrm{MeV}$. From the point of view of present applications the most important are the branching ratios to the low lying states. Such branchings are the most difficult to determine experimentally, because they are often not accompanied by $\gamma$ rays. Indeed, most of the difference between the present result and the antineutrino spectra of I can be traced to the new values of ground state branching ratios in just 4 nuclei, ${ }^{90} \mathrm{Rb}$, ${ }^{92} \mathrm{Rb},{ }^{97} \mathrm{Y}$, and ${ }^{98} \mathrm{Y}$. All of them were known previously and are known now, but the new experimental data differs from the old ones.

Finally, one has to estimate the uncertainty related to our model assumptions for unknown decays. As in I we assume that the main effect is concentrated in the parameter $\alpha^{\prime}$ describing the population of states below the pairing gap. As noted above, we assume that $\alpha^{\prime}$ is $50 \%$ uncertain for $Q-P>5 \mathrm{MeV}$ and $80 \%$ uncertain for $Q-P \leq 5 \mathrm{MeV}$. All errors are, naturally, correlated and are treated as such. The resulting curve for ${ }^{235} \mathrm{U}$ is shown in Fig. 3.

The most important quantity is the total error of the calculated spectrum. The preceding discussion shows that it is still an open question as to how to calculate such an error. As a conservative estimate we assumed that for the first three categories of error sources $\frac{1}{3}$ of the errors are correlated and the rest are uncorrelated. Adding the four partial errors for each energy in quadrature we arrived at the resulting error curve in Fig. 3.

Not surprisingly the present more careful error estimate is quite close to (although slightly smaller than) the error estimate in I. It is also quite near the uncertainties of Dickens ${ }^{11}$ and much larger than the uncertainties of Avignone and Greenwood. ${ }^{6}$ The relative errors are very similar for all con-

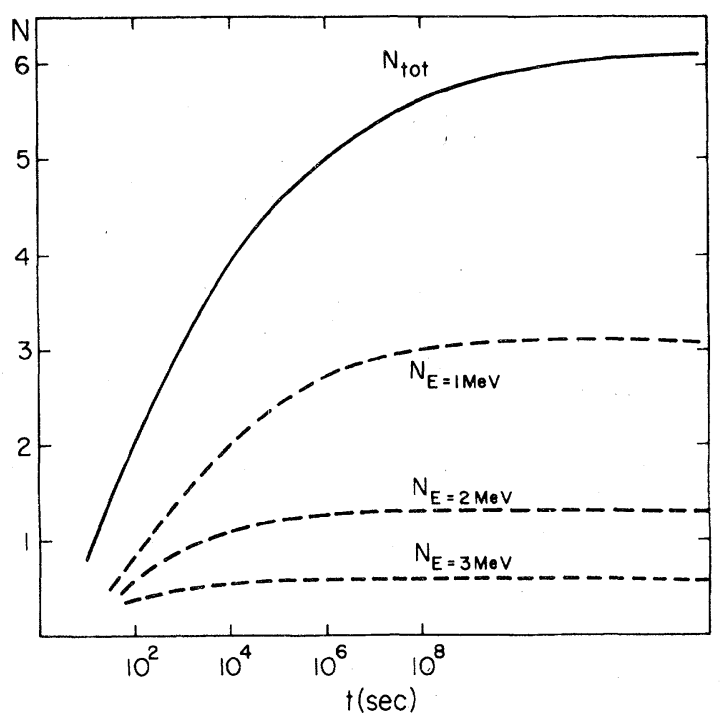

FIG. 4. Exposure time dependence of the total number of electrons per fission $\left(N_{\text {tot }}\right)$ and of the number of electrons ( $n / \mathrm{MeV}$ fission) at indicated energies. Calculation performed for ${ }^{235} \mathrm{U}$ with thermal neutron fission. 
sidered electron and antineutrino spectra. Thus Fig. 3 can be used in conjunction with Tables I and II in evaluating the absolute uncertainty of all individual spectra. At the present time it does not appear to be possible to improve substantially the accuracy of this $a b$ initio type of calculation.

The exposure time dependence of the spectrum [Eq. (11)] has been briefly discussed in I. For neutrino physics applications this is of a minor consequence because equilibrium is established very quickly at higher energies, $E \geq 3 \mathrm{MeV}$. However, the electron spectrum measurements are usually performed in a nonequilibrium situation. It is, therefore, important to take the exposure time dependence into account when comparing the experimental and calculated electron spectra. Figure 4 shows how the electron spectrum and its integral evolve with exposure time. It is seen that the total number of electrons, and the spectrum for energies less than $\sim 2 \mathrm{MeV}$ does not reach equilibrium at $10^{4}-10^{5} \mathrm{~s}$, which is a typical time used in electron spectrum measurements. The calculations of Refs. $6-10$ are based on infinite exposure time and should, therefore, overestimate the electron spectrum at low energies (and its integral).

Another way of testing our calculations is a comparison of the averaged electron and neutrino energies calculated here, with the same quantities independently obtained in Ref. 20 . In our case these quantities were evaluated as

$$
\begin{aligned}
\left\langle E_{\bar{v}}\right\rangle & =\int_{0}^{\infty} N\left(E_{\bar{v}}\right) E_{\bar{v}} d E_{\bar{v}},\left\langle T_{e}\right\rangle \\
& =\int_{m_{e} c^{c^{2}}}^{\infty} N\left(E_{\beta}\right)\left(E_{\beta}-m_{e} c^{2}\right) d E_{\beta}
\end{aligned}
$$

Owing to the energy weighing the large energies contribute relatively more to this quantity than to the total number of electrons (or antineutrinos) per fission. Using our error curve (Fig. 3) we assign an uncertainty of $7 \%$ to these quantities. According to Ref. 20 the average energies $\left\langle E_{\bar{v}}\right\rangle$ (and $\left\langle T_{e}\right\rangle$ ) are equal to $8.68 \pm 0.06(6.43 \pm 0.05)$ for ${ }^{235} \mathrm{U}$ (thermal), $11.14 \pm 0.11(8.25 \pm 0.08)$ for ${ }^{238} \mathrm{U}$ (fash fission), $7.15 \pm 0.11(5.30 \pm 0.10)$ for ${ }^{239} \mathrm{Pu}$ (thermal), and $8.78 \pm 0.09(6.51 \pm 0.07)$ for ${ }^{241} \mathrm{Pu}$ (thermal), in perfect agreement with the corresponding values of Table $I$.

The electron and antineutrino spectra can be affected by neutron captures on fission fragments and on the reactor structural material. The latter usually does not lead to electron emitters with large $Q$ values with a possible exception of $\mathrm{Al}$. The neutron capture cross section on ${ }^{27} \mathrm{Al}$ is, however, only $\sigma_{c}=0.23 \mathrm{~b}$ and thus relatively little activation takes place. The effect of neutron captures of fission products prior to their beta decay could be estimated in a following way. Let $N$ be the number of atoms of the isotope $Z, A$. The number of beta decays per second of this isotope is

$$
\eta_{\beta}=N / \tau,
$$

where $\tau$ is the beta decay mean lifetime. The number of neutron captures is

$$
\eta_{c}=F \cdot \sigma \cdot N
$$

where $F$ is the neutron flux and $\sigma$ is the neutron capture cross section. It is easy to see that $\eta_{\beta}$ exceeds $\eta_{c}$ by eight orders of magnitude for fluxes $F \sim 10^{14} / \mathrm{cm}^{2} \mathrm{~s}$, cross sections $\sigma \sim 1 \mathrm{~b}$, and lifetimes $\tau \sim 10^{2} \mathrm{~s}$. Thus neutron captures should be negligible for short lived, high $Q$ value fission fragments of primary interest here.

We have performed calculations of the $\bar{v}$ spectrum associated with the thermal neutron fission of ${ }^{235} \mathrm{U}$ corresponding to $300 \mathrm{~d}$ exposure time and neutron fluxes $\phi=10^{2}, 3 \times 10^{13}, 10^{15}$ $n / \mathrm{cm}^{2} \mathrm{~s}$. The three spectra agree with each other

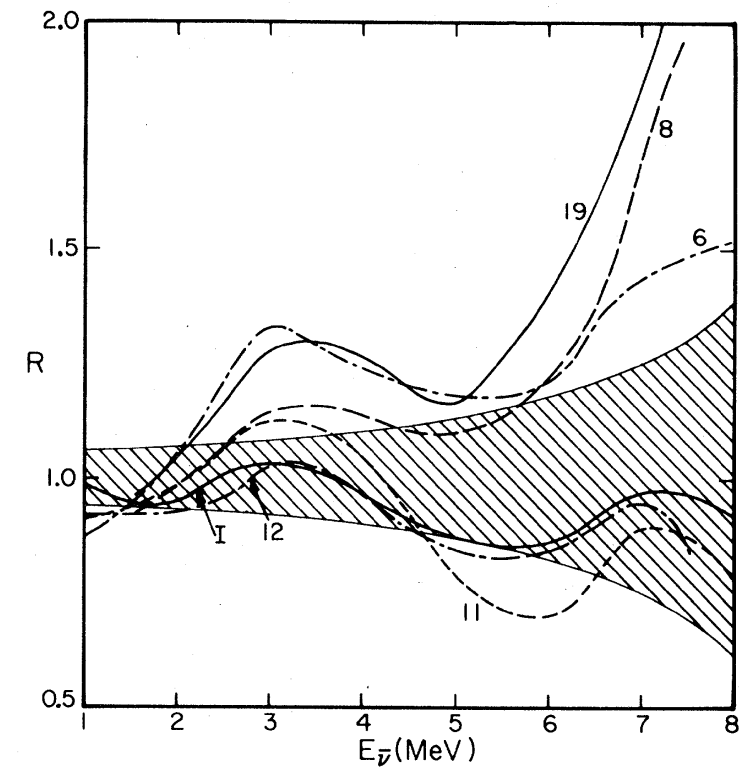

FIG. 5. Comparison of various recently calculated $\bar{v}$ spectra associated with ${ }^{235} \mathrm{U}$ fission. Each curve is labeled by the corresponding reference number. The plotted quantity $R$ is the ratio between the number of antineutrinos per $\mathrm{MeV}$ calculated there and in the present work. The shaded area is the allowed region (one standard deviation) using the present error assignment. 
with accuracy better than $1 \%$, confirming our estimate that the neutron captures on fission fragments affect negligibly the antineutrino and electron spectra above $1 \mathrm{MeV}$.

In Fig. 5 we compare the antineutrino spectra calculated recently by various authors. Very crudely they can be divided into two groups; the "soft" spectra (present work, I, and Refs. 11 and 12) and the "hard" spectra (Refs. 6, 8, and 19). The differences well within the error bars, between the present result and I are caused primarily, as pointed out above, by new experimental data in a very few known nuclei. The spectrum of Dickens ${ }^{11}$ is even softer than the spectrum of I. At least part of the difference can be attributed to the approximation adopted in Ref. 11, which results in an underestimate of the high energy part of the spectrum.

The other spectra are considerably harder. One source of this discrepancy is certainly the prescription for treatment of the unknown decays. However, the difference is already noticeable at $3-4 \mathrm{MeV}$ and is probably also related to some differences in the input data. Due to the vast amount of such input data a detailed comparison is very difficult.

\section{RELATIONSHIP BETWEEN REACTOR ELECTRON AND ANTINEUTRINO SPECTRA}

Both spectra are obviously determined by the same set of nuclear parameters and either of them can be used as a testing ground of calculations. A successful theory must predict both spectra equally well.

In Ref. 12 the different measured electron spectra are compared to each other and to the calculations of $I$ and Ref. 7. The agreement with $I$ is very good, while the electron spectrum of Ref. 7 is considerably higher than the experimental one. The present electron spectrum of ${ }^{235} \mathrm{U}$ is somewhat harder than the experimental one, ${ }^{12}$ but it still agrees within error bars at all energies up to $E_{e}=7.5 \mathrm{MeV}$.

Other aspects of the electron spectrum are discussed in Ref. 11. It is shown there that calculations reproduce the decay time dependence of the average beta energy as well as many of its spectral properties. The discrepancy between the results of Tsoulfanides et $a l^{21}$ and the Oak Ridge results ${ }^{11}$ is also apparent; the former are systematically higher at short cooling times. A similar trend is noticed in Ref. 12.

The empirical procedure of converting the electron spectrum directly into the antineutrino spec- trum, without the summation of Eq. (1), has been developed by Carter $e t a l .^{22}$ and further analyzed in I. This procedure, estimated in I to be about $10 \%$ accurate, has three difficulties. In order to evaluate Eq. (8) of I, one has to take a third derivative of the electron spectrum. This is very difficult for a function known only in a tabular form and, therefore, in I we approximated the electron spectrum by an analytic function. However, the errors of the fit then contribute to the overall error. Furthermore, in order to find the neutrino spectrum at an energy $E_{\bar{v}}$ we have to know the electron spectrum (or its third derivative) at energies up to about $E_{\bar{v}}+3 \mathrm{MeV}$. Thus some extrapolation is necessary for larger $E_{\bar{v}}$ values. Thirdly, the final result depends to some, although slight, degree on the averaging over the atomic number $Z$. All these difficulties were included in the uncertainty estimate of $I$.

Another conversion procedure has been adopted in Ref. 12. Although conceptually similar to that used in I, it eliminates the problem of the third derivative and reduces to some extent the problem of extrapolation.

An even simpler approach has been suggested by Hahn. ${ }^{23}$ It is based on the observation that for energies considerably larger than the electron mass (in practice $E \geq 3 \mathrm{MeV}$ ) the electron and antineutrino spectra have very similar shapes. They differ by the Coulomb correction factor, which is less important at large energies. To compensate for the Coulomb effect one has to shift the electron spectrum by the amount $E_{\mathrm{sh}}$, that is

$$
N_{e}\left(E-E_{\mathrm{sh}}\right)=N_{\bar{v}}(E) .
$$

It turns out that the shift $E_{\mathrm{sh}}$ lies in the interval $0-100 \mathrm{keV}$ for all considered spectra and all energies $3 \leq E \leq 8 \mathrm{MeV}$. In Fig. 6 we show the error associated with such a procedure, that is, the quantity

$$
\Delta=\frac{N_{\bar{v}}(E)-N_{e}\left(E-E_{\mathrm{sh}}\right)}{N_{\bar{v}}(E)}
$$

for three representative spectra and for $E_{\mathrm{sh}}=50$ $\mathrm{keV}$. This error remains within $6 \%$ when the largest and most uncertain energy, $E=8 \mathrm{MeV}$, is excluded. The maxima and minima of $\Delta$ are related to the fact that different $Z$ values contribute differently at different energies. To appreciate better the smallness of $\Delta$ let us note that over the considered energy interval the spectrum changes by over a factor of 100 , and the three considered spectra differ by as much as a factor of 4 . 
This simple procedure seems to work very well and should be used as an independent test of more elaborate procedures.

Thus the conversion method in any of its forms allows derivation of the associated antineutrino spectrum with an accuracy superior to the summation method used in Sec. II, provided an accurate electron spectrum is available. The good agreement between the calculated electron and $\bar{v}$ spectra of I on one hand, and the experimental electron spectrum and the converted $\bar{v}$ spectrum of Ref. 12 on the other hand, illustrates this point. Using conservatively the whole shaded region of Fig. 5 as a measure of uncertainty one can conclude that the hard spectra of Refs. 6, 8, and 19 do not correctly describe the fission process.

\section{APPLICATION TO ANTINEUTRINO-INDUCED REACTIONS}

The ultimate goal of our work is the application of the calculated spectra to the prediction of cross sections and rates of various antineutrino-induced reactions studied at nuclear reactors. In particular the agreement, or lack of it, between the predicted and measured quantities is the most straightforward test of neutrino oscillations. ${ }^{24}$

Let us first enumerate the reactions considered and briefly discuss the parameters involved.

The inverse neutron $\beta$ decay (charged current proton reaction or ccp) $\bar{v}_{e}+p \rightarrow n+e^{+}$is the reaction with the largest cross section. For monoenergetic neutrinos of reactor energies the cross section depends only on the neutron lifetime determined recently $^{25}$ with $2 \%$ accuracy. We neglect the small $(\sim 1 \%)$ radiative correction.

The charged current deuteron disintegration ced reaction $\bar{v}_{e}+d \rightarrow n+n+e^{+}$is the reaction which gave the most anomalous result, and hence evidence for neutrino oscillations, in Ref. 1. Since I was written several authors ${ }^{26,27}$ pointed out that the finite range of nuclear forces, and their incomplete charge independence, can affect the resulting cross section in a noticeable way.

Table III illustrates the effect of the various nucleon-nucleon interaction parameters on the averaged ced cross section

$$
\begin{aligned}
\bar{\sigma}_{\mathrm{ccd}}= & \int_{m_{e} c^{2}}^{\infty} d E_{e^{+}} \\
& \times \int_{B_{1}+m_{e} c^{2}}^{\infty} d E_{\bar{v}} N\left(E_{\bar{v}}\right) \frac{d \sigma}{d E_{e^{+}}}\left(E_{\bar{v}}\right),
\end{aligned}
$$

TABLE III. Effect of the nucleon-nucleon interaction parameters on the cross section for the ccd reaction (for thermal neutron fission of ${ }^{235} U$ ).

\begin{tabular}{cccc}
\hline \hline$a_{n n}(\mathrm{fm})$ & $r_{s}\left(\mathrm{MeV}^{-2}\right)$ & $r_{t}\left(\mathrm{MeV}^{-1}\right)$ & $\bar{\sigma} \cdot\left(10^{-44} \mathrm{~cm}^{2} /\right.$ fission $)$ \\
\hline-18.5 & $1.4 \times 10^{-2}$ & $8.8 \times 10^{-3}$ & 1.13 \\
-18.5 & 0 & 0 & 1.18 \\
-23.7 & 0 & 0 & 1.27 \\
-16.0 & $1.4 \times 10^{-2}$ & $8.8 \times 10^{-3}$ & 1.09 \\
\hline
\end{tabular}

where the differential cross section is taken from Ref. 28 and $B_{1}$ is the reaction threshold ( $B_{1}$ $=4.1 \mathrm{MeV}$ ). Note that the value $a_{n n}=-18.5$ fm seems to be the best estimate of the neutronneutron scattering length available at the present time. ${ }^{29}$ Other effects, notably the meson exchange current effects, can change the cross section (11) by as much as $5 \% .^{30}$

The next reaction is the neutral current deuteron (ncd) disintegration $\bar{v}+d \rightarrow n+p+\bar{v}$. The cross section formula can also be found in Ref. 28. The finite range of the neutron-proton interaction has very little effect and thus we simply used the zero range approximation. Note that the cross section depends, as in the case of the ccd reaction, only on the axial vector coupling constant and is, therefore, independent of the Weinberg angle (as long as the standard model is used).

The ccp and ccd reactions measure directly the

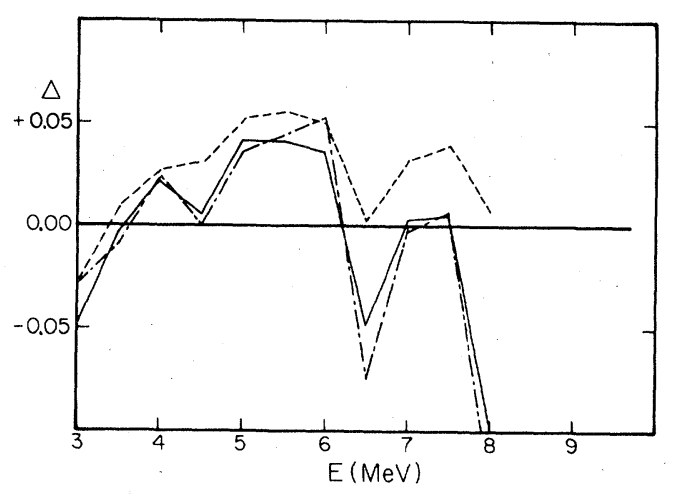

FIG. 6. Relation between the electron and antineutrino spectra. The quantity $\Delta$ is the relative difference between the antineutrino spectra and the shifted electron spectrum [see Eq. (10)]. The full curve is for ${ }^{235} \mathrm{U}$ (thermal neutrons), dashed curve is for ${ }^{238} \mathrm{U}(0.5 \mathrm{MeV}$ neutrons), and the dot and dashed curve is for ${ }^{239} \mathrm{Pu}$ (thermal neutrons). 
number of electron antineutrinos of a given energy. On the other hand the ncd reaction has the same cross section for antineutrinos of an arbitrary flavor. Consequently it should not be affected by the neutrino oscillations and may be used as a monitor of the antineutrino flux.

The last reaction to consider is the antineutrino electron scattering. Both the charged current and neutral currents contribute to this reaction; in fact in the standard model of weak interactions they destructively interfere. ${ }^{31}$ The differential cross section for monoenergetic neutrinos has been given in I. In Table IV we show the total cross section integrated over the electron kinetic energies from $T_{1}=1 \mathrm{MeV}$ to $T_{2}=6 \mathrm{MeV}$ and for the standard value $\sin ^{2} \theta_{w}=0.25$. More detailed cross sections could be calulated using the spectra of Table I or can be obtained from the authors on request.

The averaged cross sections for all these reactions and for all considered nuclear fuels are shown in Table IV. Because we use a standard relative error curve, Fig. 3, the relative errors for all fission materials are very similar and only one is given (last column). Also shown in Table IV is the ratio of ccd/ncd cross sections used in Ref. 1. This ratio is indeed remarkably insensitive to the composition of the reactor fuel.

In some reactor neutrino experiments the fuel composition can change appreciably during the long measuring time. Thus the corresponding antineutrino spectrum will also become time dependent. It should be noted that this is a second order effect, depending only on the yield part of the data. Provided the reactor fuel composition at any given time is known, one can use the spectra of Table I or the averaged cross sections of Table IV and compute the resulting spectrum, or the resulting cross section. As an example let us take a power reactor which immediately after refueling derives $95 \%$ of its power from ${ }^{235} \mathrm{U}$ fission by thermal and epithermal neutrons, and $5 \%$ of its power from ${ }^{238} \mathrm{U}$ fast neutron fission. After roughly a year of operation $63 \%$ of its power comes from ${ }^{235} \mathrm{U}, 6.5 \%$ from ${ }^{238} \mathrm{U}$ fission, $28 \%$ from ${ }^{239} \mathrm{Pu}$ fission, and $2.5 \%$ from ${ }^{241} \mathrm{Pu}$ fission. The average cross section decreases then by the following amounts: $10 \%$ (ccp), $17 \%$ (ccd), and $14 \%$ (ncd). Such changes are clearly noticeable but small enough so that the reactor neutrino experiments can be performed in an essentially model independent way.

\section{SUMMARY}

In this update of our previous work we have calculated the antineutrino and electron spectra associated with a variety of fissioning nuclei and different incident neutron energies. The obtained set of spectra allows us to compute the antineutrino spectrum of essentially any nuclear reactor at an arbitrary time in its refueling cycle.

Our results show that for a given actinide nucleus the spectrum depends only slightly on the incident neutron energy. The changes from one nucleus to another are, however, quite substantial. Nevertheless, typical average cross sections of an antineutrino induced reaction will change by only $10-15 \%$ per year due to the changing reactor fueld composition.

TABLE IV. Averaged, cross sections in $10^{-44} \mathrm{~cm}^{2} /$ fission and their relative uncertainty (last column).

\begin{tabular}{|c|c|c|c|c|c|c|c|c|c|c|c|}
\hline $\begin{array}{l}\text { Nucleus } \\
n \text { energy }\end{array}$ & $(\mathrm{MeV})$ & $\begin{array}{l}{ }^{235} \mathrm{U} \\
\text { ther }\end{array}$ & $\begin{array}{c}{ }^{235} \mathrm{U} \\
0.5\end{array}$ & $\begin{array}{c}{ }^{235} \mathbf{U} \\
14\end{array}$ & $\begin{array}{c}{ }^{238} \mathrm{U} \\
0.5\end{array}$ & $\begin{array}{c}{ }^{238} \mathrm{U} \\
14\end{array}$ & $\begin{array}{c}{ }^{239} \mathrm{Pu} \\
\text { ther }\end{array}$ & $\begin{array}{c}{ }^{239} \mathrm{Pu} \\
0.5\end{array}$ & $\begin{array}{c}{ }^{240} \mathrm{Pu} \\
0.5\end{array}$ & $\begin{array}{l}{ }^{241} \mathrm{Pu}_{\mathrm{u}} \\
\text { ther }\end{array}$ & $\begin{array}{l}\Delta^{\mathrm{e}} \\
(\%)\end{array}$ \\
\hline \multicolumn{12}{|c|}{ Reaction } \\
\hline & ccp & 63.4 & 64.2 & 47.8 & 88.5 & 77.9 & 43.9 & 45.0 & 51.0 & 63.2 & 13 \\
\hline & $\operatorname{ccd}^{\mathrm{a}}$ & 1.13 & 1.19 & 0.83 & 1.78 & 1.61 & 0.63 & 0.67 & 0.77 & 1.04 & 21 \\
\hline & $\mathrm{ncd}^{\mathrm{d}}$ & 3.10 & 3.17 & 2.29 & 4.55 & 4.03 & 1.94 & 2.02 & 2.29 & 2.97 & 17 \\
\hline & el. $s c^{b}$ & 0.37 & 0.38 & 0.29 & 0.50 & 0.44 & 0.28 & 0.28 & 0.32 & 0.38 & 11 \\
\hline & $R^{\mathrm{c}}$ & 0.36 & 0.37 & 0.36 & 0.39 & 0.40 & 0.32 & 0.33 & 0.34 & 0.35 & 5 \\
\hline
\end{tabular}

${ }^{\mathrm{a} C a l c u l a t e d}$ with the following parameters: $a_{n n}=-18.5 \mathrm{fm}, r_{s}=1.4 \times 10^{-2} \mathrm{MeV}^{-1}, r_{t}=8.8 \times 10^{-3} \mathrm{MeV}^{-1}$. bIntegrated over electron energies $1-6 \mathrm{MeV}$ "standard model" $\sin ^{2} \theta_{2}=0.25$.

${ }^{\mathrm{c}} \boldsymbol{R}=\sigma(\mathrm{ccd}) / \sigma(\mathrm{ncd})$.

${ }^{\mathrm{d}}$ Calculated with $a_{n p}=-23.7 \mathrm{fm}$, zero range.

'Relative error of average cross section, identical for all considered nuclei, see text. 
The analysis of uncertainties associated with the spectrum has been also substantially improved. The uncertainties are related to a variety of causes, but ultimately all of them are caused by the short lifetimes and small fission yields of the fission products with large beta decay $Q$ values. Large reduction of these uncertainties does not seem feasible at the present time.

However, the prospects of a direct conversion of the experimentally determined electron spectrum into the antineutrino spectrum are very promising. It seems that accuracies $\sim 5 \%$ could be achieved.

\section{ACKNOWLEDGMENTS}

The enlightening discussion with members of the Caltech group, particularly with F. Boehm, A.

Hahn, H. Kwon, and J. L. Vuilleumier are appreciated. This work was supported by DOE Grants No. Ey-76-C-03-0063 and DE-AC14-76FF02170.
${ }^{1}$ F. Reines, H. W. Sobel, and E. Pasierb, Phys. Rev. Lett. 45, 1307 (1980).

${ }^{2}$ F. Boehm et al., Phys. Lett. 97B, 310 (1980); H. Kwon et al., Phys. Rev. D (to be published).

${ }^{3}$ V. S. Kozik et al., Yad. Fiz. 32, 301 (1980); V. A. Lubimov et al., Phys. Lett. 94B, 266 (1980).

${ }^{4}$ A. De Rujula et al., Nucl. Phys. B168, 54 (1980).

${ }^{5}$ B. R. Davis, P. Vogel, F. M. Mann, and R. E. Schenter, Phys. Rev. C 19, 2259 (1979).

${ }^{6}$ F. T. Avignone III and Z. D. Greenwood, Phys. Rev. C 22, 594 (1980).

${ }^{7}$ F. T. Avignone III, L. P. Hopkins, and Z. D. Greenwood, Nucl. Sci. Eng. 72, 216 (1979).

${ }^{8}$ A. A. Borovoi, Yu. L. Dobrynin, and V. I. Kopeikin, Yad. Fiz. 25, 264 (1977).

${ }^{9}$ S. A. Fayans, L. A. Mikaelyas, and Y. L. Dobrynin, J. Phys. G 5, 209 (1979).

${ }^{10}$ V. I. Kopeikin, Yad. Fiz. 32, 62 (1980).

11 J. K. Dickens, Phys. Rev. Lett. 46, 1061 (1981).

${ }^{12}$ K. Schreckenbach et al., Phys. Lett. 99B, 25 (1981).

${ }^{13}$ Fission Product Library of the Evaluated Nuclear Data File, Version V. Available from, and maintained by, the National Nuclear Data Center at the Brookhaven National Laboratory.

${ }^{14}$ A. H. Wapstra and K. Bos, At. Data Nucl. Data Tables 19, 177 (1977).

${ }^{15}$ A. H. Wapstra and K. Bos, At. Data Nucl. Data Tables 17, 474 (1976).

${ }^{16}$ K. Aleklett, G. Nyman, and G. Rudstam, Nucl. Phys. A246, 425 (1976).
${ }^{17}$ H. V. Klapdor et al., Z. Phys. A $\underline{299}, 213$ (1981); Phys. Rev. C 23, 1269 (1981).

${ }^{18}$ M. Epherre et al., Phys. Rev. C 19, 1504 (1979).

${ }^{19}$ F. T. Avignone III and L. P. Hopkins, in Neutrino78, Proceedings of the International Conference on Neutrino Physics and Astrophysics, edited by E. C. Fowler (Purdue University Press, Lafayette, Indiana 1978), p.C42.

${ }^{20} \mathrm{R}$. Sher et al., Stanford University report, 1976 (unpublished).

${ }^{21}$ N. Tsoulfanides, B. W. Wehring, and N. E. Wyman, Nucl. Sci. Eng. 43, 42 (1971).

${ }^{22}$ R. E. Carter, F. Reines, J. J. Wagner, and N. E. Wyman, Phys. Rev. 113, 280 (1959).

${ }^{23}$ A. Hahn, ILL report, 1980 (unpublished).

${ }^{24} \mathrm{P}$. Vogel, Proceedings of the Neutrino Mass Miniconference, Telemark, Wisconsin, edited by V. Barger and D. Cline, University of Wisconsin Report 186, 1981.

25J. Bryne et al., Phys. Lett. 92B, 274 (1980).

${ }^{26}$ A. Dar, Technion Report PH-80-40, 1980 (unpublished).

${ }^{27}$ W. R. Gibbs and G. J. Stephenson, Phys. Rev. D $\underline{23}$, 260 (1981).

${ }^{28}$ T. Ahrens and T. P. Lang, Phys. Rev. C 3, 979 (1971).

${ }^{29}$ B. Gabioud et al., Phys. Rev. Lett. 42, 1508 (1979).

30J. Hosek and E. Truhlik, Phys. Rev. C 23, 665 (1981).

${ }^{31}$ B. Kayser, E. Fischbach, S. P. Rosen, and H. Spivack, Phys. Rev. D 20, 87 (1979). 